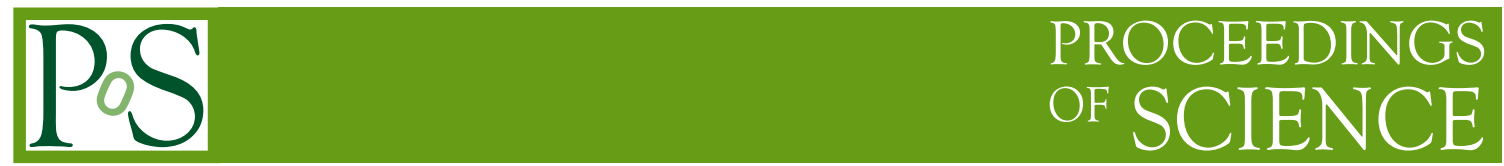

\title{
QCD at non-zero temperature from the lattice
}

\author{
Harvey B. Meyer* \\ PRISMA Cluster of Excellence, Institut für Kernphysik and Helmholtz Institut Mainz, \\ Johannes Gutenberg-Universität Mainz, D-55099 Mainz, Germany \\ E-mail: meyerh@kph.uni-mainz.de
}

I review the status of lattice QCD calculations at non-zero temperature. After summarizing what is known about the equilibrium properties of strongly interacting matter, I discuss in more detail recent results concerning the quark-mass dependence of the thermal phase transition and the status of calculations of non-equilibrium properties.

The 33rd International Symposium on Lattice Field Theory

14 - 18 July 2015

Kobe International Conference Center, Kobe, Japan*

${ }^{*}$ Speaker. 


\section{Introduction}

In the 1960's, bulk hadronic matter was predicted to undergo a thermal phase transition due to the high multiplicity of hadrons [1]. With the advent of QCD as the theory of the strong interaction, the phase above that transition was identified with a weakly interacting gas of quarks and gluons [2] in virtue of asymptotic freedom. High-temperature QCD was studied more systematically starting in the late 1970's [3]. The first studies of strongly interacting matter at non-zero temperature $T$ using lattice QCD date back to 1981 [4]. Lattice QCD is so far the only computational framework to have led to reliable quantitative predictions on equilibrium quantities such as the equation of state, the transition temperature $T_{c}$ and quark number susceptibilities in the temperature range $100 \lesssim$ $T / \mathrm{MeV} \lesssim 500$. Thereby it has had an important impact on heavy-ion collision phenomenology. Lattice QCD has also contributed decisively to answering qualitative questions such as 'What is the nature of the thermal transition?', 'What is the character of the high-temperature phase at $T=$ $250 \mathrm{MeV}, 500 \mathrm{MeV}$ and $1000 \mathrm{MeV}$ ?' or 'What is the character of the low-temperature phase at $T=$ $140 \mathrm{MeV}$ ?'. While it is accepted that the medium is a gas of pions a sufficiently low-temperatures, and a gas of quarks and gluons at sufficiently high temperatures, lattice methods are the only theory methods able to bridge the gap between these two limits.

I attempt to review the status of the lattice QCD effort. The achievements are remarkable, yet there are still significant challenges to be overcome before we can claim that all the qualitative questions formulated above are answered in a satisfactory manner, particularly concerning the nonequilibrium quantities. A review of this length cannot possibly be exhaustive. Fortunately, several reviews have appeared in recent years $[5,6,7,8]$, as well as lattice conference write-ups.

The next section gives an overview of what has been learnt about QCD at non-zero temperature from the lattice; here the results are not necessarily recent. In section 3, I cover recent work on the quark-mass dependence of the phase diagram (Columbia plot). Section 4 is devoted to nonequilibrium quantities. The last section contains some final remarks.

\section{QCD at non-zero temperature: a portrait}

We begin by fixing some notation. Lattice QCD employs the Matsubara formalism of thermal field theory, where the partition function $Z \equiv \operatorname{Tr}\left\{e^{-\beta \hat{H}}\right\}$ is represented as a Euclidean path integral, in which bosons have periodic, fermions antiperiodic boundary conditions in time [9]. The extent of the time and space directions is $\beta=1 / T=N_{t} a$ and $L$ respectively. We will only consider isospin symmetric QCD, with up-down quark masses $m_{u d}$ and strange quark mass $m_{s}$. The isospin Pauli matrices are denoted $\tau^{a}$ and $\psi$ is the up/down quark doublet field. Let $\langle\bar{\psi} \psi\rangle$ be the chiral condensate. In the chiral limit, it is the order parameter for chiral symmetry breaking: it vanishes above the chiral transition. Its susceptibility can be written $\chi_{m}=\frac{T}{L^{3}} \frac{\partial^{2}}{\partial m_{\mathrm{ud}}^{2}} \log Z$. Thermodynamic quantities of interest include the energy density $e$, pressure $p$ and entropy density $s$. Quark number susceptibilities can be written as $\chi_{s}=\beta \int d^{3} x\left\langle J_{0}(x) J_{0}(0)\right\rangle\left(x_{0} \neq 0\right)$, if $J_{\mu}$ is a conserved vector current. We will address a few transport coefficients, such as the diffusion coefficient $D$ and the shear viscosity $\eta$.

\subsection{Properties at physical $u, d, s$ quark masses}

The transition temperature.- The most thorough investigations of the transition temperature have 
been performed with staggered fermions. The BW collaboration [10] locates the transition temperature at $155(2)(3) \mathrm{MeV}$, based on the inflection point of $m_{u d}\left(\left.\langle\bar{\psi} \psi\rangle\right|_{0} ^{T}\right) / T^{4}$. As for results based on the peak of the chiral susceptibility, the same publication [10] gives $T_{c}=147(2)(3) \mathrm{MeV}$, while the HotQCD collaboration [11] obtained $T_{c}=154(8)(1) \mathrm{MeV}$. In the latter calculation, an additional feature was the use of the expected scaling laws near the chiral limit.

Last year, a calculation [12] using domain-wall fermions yielded the result 155(1)(8)MeV based on the same quantity, thus providing a universality test, even though this calculation was performed at a single, fixed value of $N_{t}=8$. Calculations with Wilson fermions are progressively moving toward physical quark masses [13].

Deconfinement.- It is an intriguing question whether the deconfinement of partons occurs at the same temperature as the chiral transition. Within QCD, the question cannot be given a completely sharp meaning, since the transition is a crossover [14, 12], and there is no known strict order parameter for confinement. Nevertheless, the light-quark number susceptibility $\chi_{s}^{u, d} / T^{2}$ admits an inflection point practically at the same temperature as the chiral restoration $[15,16]$. The rise of the strangeness fluctuations as a function of temperature is delayed by about $20 \mathrm{MeV}$. Ratios of cumulants of the baryon number $R_{42}^{B}=\chi_{4}^{B} / \chi_{2}^{B}$, which are identically unity in the hadron-resonance gas model, provide another useful criterion. The ratio $R_{42}^{B}$ deviates substantially from unity already at $T=155 \mathrm{MeV}$ [17].

Thermodynamic potentials. - Good agreement was reached between the calculations of the BW [18] and the HotQCD [19] collaborations once the continuum limit was taken based on the ranges $6 \leq N_{t} \leq 16$ and $6 \leq N_{t} \leq 12$ respectively. At $T=255 \mathrm{MeV}$, that is, $100 \mathrm{MeV}$ above the chiral transition, the pressure amounts to about half the Stefan-Boltzmann pressure corresponding to free quarks and gluons. And, the ratio ${ }^{1}(e-3 p) /\left[\frac{3}{4}(e+p)\right]$ evaluates to about a third. There are thus large deviations from both a weakly-coupled regime and from a scale-invariant regime.

The hadron resonance gas (HRG) model vs. lattice data.- The HRG model assumes that the thermal medium consists of quasiparticles with a spectrum identical to the hadron spectrum in vacuum. It is justified to some extent by the studies showing that resonances whose width is narrow compared to the temperature can be treated as constituents of the medium [20]. The model describes the thermodynamic potentials and the quark number susceptibilities very well up to $T=$ $155 \mathrm{MeV}$. The level of agreement is surprisingly good, given that most of the $T=0$ resonances are not narrow compared to the temperature and that thermal effects are bound to modify the spectrum of thermal quasiparticles.

Static screening masses. - In the high-temperature phase, a number of static correlation lengths (=inverse screening masses) have been computed. The light-quark isovector masses are expected to approach $2 \pi T$ at high temperatures. This behavior is approximately observed in several calculations [21, 22, 23, 24, 25]. However, looking more closely, the weak-coupling correction is of order $g^{2}(T)$, with a positive coefficient [26]. What several lattice calculations have found [21, 22, 23, 24, 25 ] is that the pseudoscalar screening mass lies well below $2 \pi T$ at least until $T=500 \mathrm{MeV}$; higher temperatures have not been explored much. Also, the transverse vector screening mass is found to be below $2 \pi T$ at $T=255 \mathrm{MeV}$ [27]. Therefore, from the point of view where the $z$ direction is

\footnotetext{
${ }^{1}$ This quantity corresponds to the ratio of the expectation value of the trace of the energy-momentum tensor and the 00-component of its traceless part.
} 

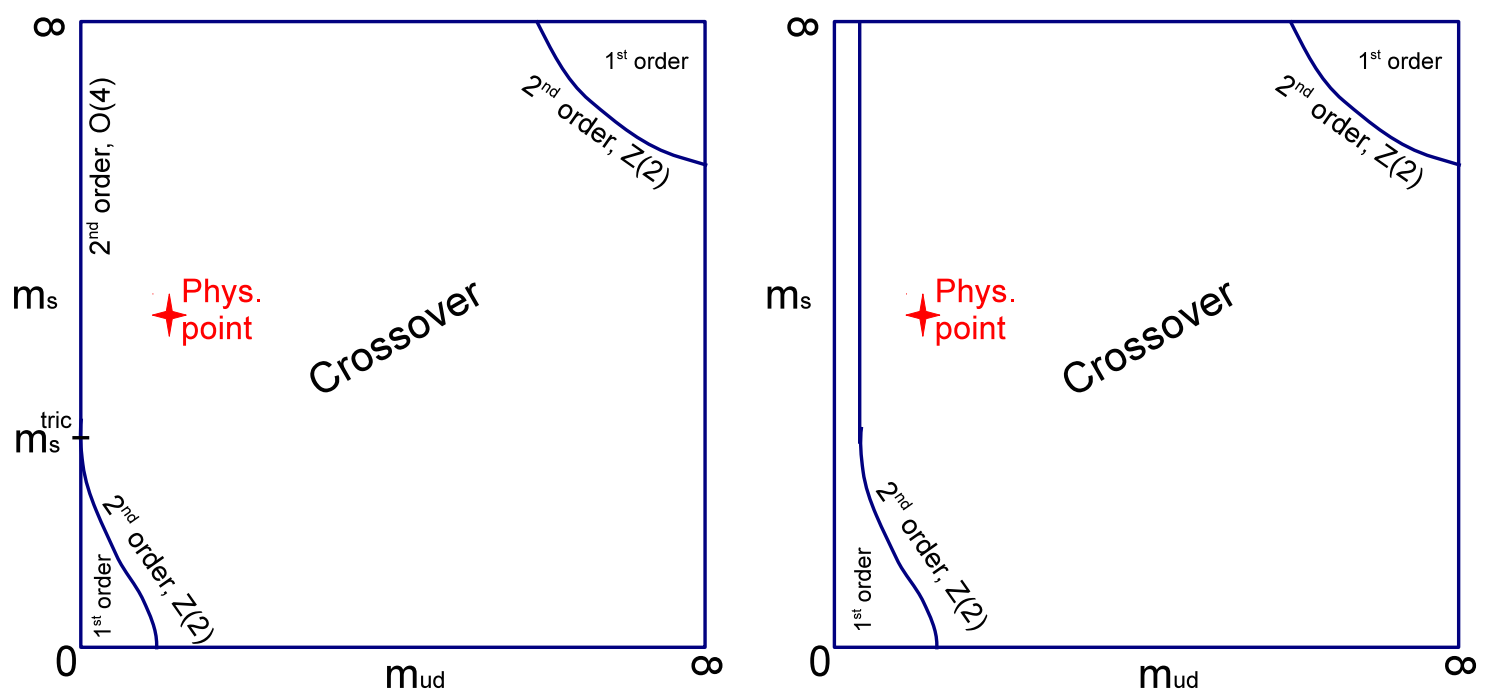

Figure 1: The 'Columbia plot', which indicates the dependence of the nature of the thermal QCD transition on the up, down and strange quark masses $m_{u d} \equiv m_{u}=m_{d}$ and $m_{s}$. Two scenarios are depicted, which differ qualitatively in the $m_{u d} \rightarrow 0$ limit. In the left, the phase transition is of second order at $m_{u d}=0$. In the right panel, the phase transition is of first order below a critical $m_{u d}$ mass.

interpreted as a Euclidean time, quarks appears to be more strongly bound than the weak-coupling treatment predicts. Finally, we remark that calculations in the low-temperature phase indicate that the pseudoscalar correlation length starts to become shorter well below $T_{c}$, see e.g. [28].

\subsection{Dependence of the phase transition on the matter content of the gauge theory}

The simplest deformation of QCD with $u, d, s$ quarks consists in varying the masses of these quarks. The nature of the thermal transition is expected to have a specific dependence on these masses, which is represented graphically in the 'Columbia plot'; see Fig. 1.

Two features are firmly established, both by universality arguments and by direct Monte-Carlo simulations. In the limit of infinite quark masses, the theory reduces to the $\mathrm{SU}(3)$ pure gauge theory, and the Z(3) symmetry associated with the center of the gauge group becomes exact. As anticipated [29], based on the fact that Z(3) invariant theories in three dimensions have first order phase transitions, the deconfinement transition is found to be first order. At the opposite corner of the Columbia plot, i.e. in the limit where $m_{u d} \rightarrow 0$ and $m_{s} \rightarrow 0$, a sharp phase transition, now associated with chiral symmetry, takes place. There are strong arguments that it is first order [30].

In a broad range of $m_{u d}$ and $m_{s}$, including the physical values of the masses, the thermal transition is a crossover. The $\mathrm{Z}(2)$ critical line at large quark masses, where the first order transition turns into a crossover, has been studied on the lattice. Pinning down the $\mathrm{Z}(2)$ critical line in the lower left corner of the diagram is the subject of current simulations; see section 3.

In the SU(2) chiral limit, i.e. when $m_{u d} \rightarrow 0$ with the strange quark mass held fixed at its physical value, the chiral transition must be a well-defined phase transition. The transition may be of second order in the $\mathrm{O}(4)$ universality class, or it may be of first order [30]. Which scenario is realized in Fig. 1 is also the subject of current calculations; see section 3. 


\subsection{Transition temperature as a function of the matter content}

The crossover temperature at physical $u, d, s$ quark masses $T_{c} \approx 155 \mathrm{MeV}$ is lower than educated guesses made in the 1980's [31]. In the following we give an overview of the dependence of the transition temperature on the matter content. For this purpose, we compare the $T_{c}$ 's of different theories in units of the Sommer reference scale $r_{0}$. However, since $\mathrm{MeV}$ units are more familiar, we set $r_{0} \doteq 0.5 \mathrm{fm}$ to quote results in $\mathrm{MeV}$.

Within the three-flavor theory, extensive calculations [13] with Wilson fermions show that the transition temperature decreases as a function of the light-quark masses. With two flavors, results have been obtained recently with Wilson fermions, both with and without a twisted-mass action. At a quark mass corresponding to a zero-temperature pion mass of about $300 \mathrm{MeV}$, the crossover is very broad. Ref. [24] quotes $T_{c}=211(5) \mathrm{MeV}$, while the result of Ref. [32] corresponds to $T_{c}=180(12) \mathrm{MeV}$ in our chosen scale setting. There is therefore some tension between these two results. The first was obtained at finite $N_{t}=16$, the second is a continuum-extrapolated result based on $N_{t} \leq 12$.

When all three quark masses become heavy, one reaches the SU(3) pure gauge theory. A recent, accurate result for the deconfinement temperature is $T_{c}=294(2) \mathrm{MeV}$ [33]. In view of these results, one may wonder if the transition temperature would decrease even further if light matter fields were added. This question has arisen in the context of walking gauge theories. It has been conjectured that the transition temperature goes all the way down to zero as the number of flavors is increased; see the results and the discussion in [34]. Once $N_{\mathrm{f}}$ is large enough for the transition to occur at $T=0$, the infrared properties of the $T=0$ system are described by a conformally invariant theory. The phase transition has been used as a tool to study gauge theories with many matter degrees of freedom, since it is not easy to numerically distinguish a chirally broken phase from the conformally invariant phase; see for instance [35].

\subsection{Gauge group dependence of the equilibrium properties}

Another direction in which one can depart from QCD is by changing the gauge group. Varying the number of colors $N_{c}$, several studies of the thermodynamic potentials of $\mathrm{SU}\left(N_{c}\right)$ gauge theory have been performed [36]. In the high-temperature phase, it is useful to define the function

$$
\bar{p}(x)=\left(\frac{p}{p_{\mathrm{SB}}}\right)\left(T=x T_{c}\right),
$$

whose value is the pressure normalized by the Stefan-Boltzmann pressure, as a function of the temperature in units of the transition temperature. The result is that within the small uncertainties, $\bar{p}$ for $3 \leq N_{c} \leq 8$ is found to be 'universal' for $x>1.1$. This empirical fact indicates that the multiplicity of the degrees of freedom scales with $\left(N_{c}^{2}-1\right)$, as expected in the weak-coupling regime. The departure from unity is still large, for instance $\bar{p}(1.6) \approx 0.5$, a value very similar to QCD with physical $u, d, s$ quark masses.

Studies have also been performed with other gauge groups, most notably with the exceptional group $\mathrm{G}(2)[37,38]$. The theory admits color singlet asymptotic states, but has no center symmetry. Remarkably, here too $\bar{p}(x)$ for $x \geq 1.1$ is consistent with the function obtained for the $\mathrm{SU}\left(N_{c}\right)$ groups [38]. 
In the low-temperature phase, it is interesting to ask whether a 'hadron' resonance gas model accounts for the equilibrium properties as well as for realistic QCD. According to the large- $N_{c}$ counting rules, interactions among the 'hadrons' should be of order $1 / N_{c}^{2}$ in the pure $\mathrm{SU}\left(N_{c}\right)$ gauge theories, and therefore even more suppressed than in QCD, thus giving the model a stronger justification. While the low-lying glueball spectrum of SU(3) gauge theory is fairly well known from lattice calculations [39], it turns out that, within the model, the thermodynamic potentials receive a substantial contribution from very massive states. Assuming that the glueball spectrum extends at higher masses as predicted by the closed-string spectrum, a good description of the thermodynamic potentials computed on the lattice is obtained [40,41]. Since the spectrum is exponential (Hagedorn-type spectrum), the model prediction depend somewhat on the value of the exponent chosen and on whether the spectrum is assumed to be charge-conjugation symmetric. Nevertheless the agreement with lattice data is quite impressive.

\subsection{Very high temperatures: making contact with perturbation theory}

Since there are large deviations from the non-interacting behavior at $T=1.6 T_{c}$, one may wonder at what temperature contact is made with perturbative calculations, which for many quantities have been pushed to high orders. In computing the pressure (or the quantity $e-3 p$ ), a multiscale problem arises at very high temperatures, since a direct approach would imply subtracting the vacuum contribution non-perturbatively at the same lattice spacing. One solution [42] consists in performing the vacuum subtraction in several steps, adding and subtracting the pressure at temperatures differing by a factor two. Another approach consists in computing the enthalpy $e+p$, which does not require a vacuum subtraction. Then a renormalization factor is required, which may for instance be computed using shifted boundary conditions [43, 44, 45, 46]. Overall perturbation theory provides a good description of the lattice data for $T>5 T_{c}$ if a coefficient of the $\mathrm{O}\left(g^{6}\right)$ contribution is fitted to the highest temperatures. However, the successive terms of the perturbative series are alternating and hardly decreasing in magnitude with the order - this has been known for a long time [47].

Is the lack of apparent convergence of the perturbative series at $T<10 T_{c}$ a symptom that the nature of the medium is completely different from the weak-coupling quark-gluon plasma picture? Probably not. After all we know that the expansion is in powers of $g$, not $\alpha_{s}=\frac{g^{2}}{4 \pi}$, and that the medium has a non-perturbative sector even at arbitrarily high temperatures. Different observables are sensitive to this sector starting at different orders.

\subsection{Summary}

- In the low-temperature phase, the hadron resonance gas model provides a good description of the equilibrium properties.

- The thermal transition is a crossover.

- The chiral and deconfinement transitions essentially coincide, with $T_{c}=155 \mathrm{MeV}$ being a typical value obtained for the crossover.

- Above the transition, the multiplicity of the degrees of freedom is consistent with the number expected for quarks and gluons;

- but many equilibrium quantities are far from the weak-coupling predictions at least until $T=$

$2.5 T_{c}$. In addition, the size of the elliptic flow observed in heavy-ion collisions points to a medium 
with a very small shear viscosity to entropy density ratio in the range $T_{c}<T<2.5 T_{c}$, typical estimates being $\eta / s \approx 0.12$ at RHIC and $\eta / s \approx 0.2$ in the ALICE experiment [48]. Taken together, the last two observations indicate that, in the range of temperatures explored in heavy-ion collisions, the partonic degrees of freedom are strongly correlated.

\section{Toward a quantitative version of the Columbia plot}

- The region around the $S U(3)$ chiral limit $m_{u d}=m_{s}=0$. - Lattice calculations find that for sufficiently small $u, d, s$ quark masses the transition is first order. However, quantitatively the findings differ wildly depending on the lattice action and lattice spacing used. Generally, the observation has been that a smaller lattice spacing leads to a smaller first-order region.

Jin et al. have published [49] and reported at this conference the result of a finite-size scaling study at $N_{t}=6$ and 8, including a continuum extrapolation. They find $m_{\pi}^{\text {crit }}=304(7)(14)(7) \mathrm{MeV}$ for the critical pseudoscalar mass. This result was obtained with an $\mathrm{O}(a)$ improved Wilson fermion action and the Iwasaki gauge action. H.-T. Ding for the Bielefeld-BNL-CCNU collaboration, on the other hand, has reported $m_{\mathrm{ps}}^{\text {crit }} \lesssim 50 \mathrm{MeV}$ with the HISQ action at $N_{t}=6$, based on infinite-volume scaling with $\mathrm{Z}(2)$ exponents. The data extends impressively to $m_{\pi}=80 \mathrm{MeV}$.

In view of the contradiction between these results, there is a real need for simulations on finer lattices, $N_{t} \geq 12$, including a continuum extrapolation, to quantify the size of the first-order region. - The $S U(2)$ chiral limit at fixed, physical $m_{s}$. - The HotQCD collaboration $[50,51]$ reports on a calculation with the HISQ action at $N_{t}=6$, in which the chiral susceptibility shows no sign of a first-order phase transition, even at $m_{\pi}=80 \mathrm{MeV}$. The results thus point to the standard scenario. The observables are fitted with $\mathrm{O}(2)$ exponents rather than $\mathrm{O}(4)$ exponents, due to the taste-splitting effects of the staggered action.

- The chiral limit in the $N_{\mathrm{f}}=2$ theory.- In the limit $m_{s} \rightarrow \infty$ one reaches the two-flavor theory. Here there have been several calculations investigating the nature of the thermal phase transition in a regime as chiral as possible. $\mathrm{O}(a)$ improved Wilson fermions at $N_{t}=16$ lead to a crossover transition for $m_{\pi}=295 \mathrm{MeV}$ [24], and so do twisted mass Wilson fermions for a pion mass of $333 \mathrm{MeV}$ [32]. Using imaginary chemical potential and the predictivity of tricritical scaling, Bonati et al. found [52] a critical pion mass of more than $600 \mathrm{MeV}$ with Wilson fermions at $N_{t}=4$. Obviously, finite-lattice-spacing effects are a major issue here too. Ch. Pinke and Ch. Czaban have reported on progress in these studies at $N_{t}=4$ and $N_{t}=6$ at this conference.

The effects of the anomalous breaking of the $U_{A}(1)$ symmetry are expected to be gradually reduced as the temperature increases. A light $\eta^{\prime}$ screening mass provides a mechanism that could make the transition first order when $m_{u d} \rightarrow 0$. In my view, while it is certainly interesting to study measures of the anomalous symmetry breaking at $T \neq 0$, discriminating between the two scenarios of Fig. 1 still requires direct simulations at small $m_{u d}$.

\section{Near-equilibrium properties}

While there is hardly an alternative to lattice QCD when it comes to first-principles predictions for equilibrium properties, out-of-equilibrium aspects of QCD at non-zero temperature are only indirectly accessible on the lattice. Let $J(x)$ be a local bosonic Hermitian operator; the relation 
between the Euclidean correlator $G$ and the spectral function $\rho$ reads

$$
G\left(x_{0}, \vec{p}\right)=\int d^{3} x e^{-i \vec{p} \cdot \vec{x}}\langle J(x) J(0)\rangle=\int_{0}^{\infty} d \omega \rho(\omega, \vec{p}) K\left(x_{0}, \omega\right), \quad K\left(x_{0}, \omega\right)=\frac{\cosh \left[\omega\left(\beta / 2-x_{0}\right)\right]}{2 \pi \sinh [\omega \beta / 2]} .
$$

This equation represents an inverse problem for $\rho(\omega, \vec{p})$, given the correlator $G$. In thermal equilibrium, its (Kubo-Martin-Schwinger, KMS) relation to the Wightman correlator

$$
\rho(\omega, \vec{p})=\left(1-e^{-\beta \omega}\right) \int_{-\infty}^{\infty} d t \int d^{3} x e^{i \omega t-i \vec{p} \cdot \vec{x}} \frac{1}{Z} \operatorname{Tr}\left\{e^{-\beta \hat{H}} \hat{J}(t, \vec{x}) \hat{J}(0)\right\}
$$

shows explicitly how it encodes real-time information ${ }^{2}$. Inserting a complete set of (unit-normalized) energy eigenstates $|n\rangle$, the formal expression for the spectral function reads

$$
\rho(\omega, \vec{p})=\frac{4 \pi}{L^{3} Z} \sinh (\beta \omega / 2) \sum_{n, m}\left|\left\langle n\left|\int_{\vec{x}} e^{-i \vec{p} \cdot \vec{x}} \hat{J}(\vec{x})\right| m\right\rangle\right|^{2} e^{-\beta\left(E_{n}+E_{m}\right) / 2} \delta\left(\omega-\left(E_{n}-E_{m}\right)\right) .
$$

Such expressions are useful to prove formal relations, such as the connection with the Minkowskispace correlators. However, what we are after are the collective excitations of the medium, which are in general not related in a simple manner to the states $|n\rangle$. The collective excitations are typically poles in the frequency variable of the infinite-volume correlator, which depend on the temperature and are not related in a simple way to the $|n\rangle$. For instance, the hydrodynamic excitations associated with conserved currents have to be there. Furthermore, quasiparticles may exist in the medium: there may be a pole at $\left(\omega=\omega_{\vec{p}}\right)$ with $\operatorname{Im}\left(\omega_{\vec{p}}\right) \lesssim \operatorname{Re}\left(\omega_{\vec{p}}\right)$; in that case, $\nu_{\mathrm{g}}=\frac{d \omega_{\vec{p}}}{d|\vec{p}|}$ is its group velocity. There are media, however, with no quasiparticles.

The inverse problem is numerically ill-posed. The success of the calculation thus depends on what resolution in the frequency $\omega$ one needs to achieve. Physically, resolving the spectral function on a finer frequency scale means that one understands the real-time evolution of a particular excitation up to a longer time-scale. As an illustrative example, we consider the isoscalar vector channel with the current $J_{i}=\frac{1}{\sqrt{2}}\left(\bar{u} \gamma_{i} \bar{u}+\bar{d} \gamma_{i} d\right)$. At $T=0$, detailed information on the spectral function is provided by $e^{+} e^{-}$collider data. For $\omega<1 \mathrm{GeV}$, the spectral function is completely dominated by the $\omega$ resonance; see Fig. 2, left panel. The spectral function vanishes below the threshold $\omega=3 m_{\pi}$, and remains very small at least until $\omega=700 \mathrm{MeV}$. These features are characteristic of QCD at low energies, and reflect the confinement of quarks. Correspondingly, one expects a very different form of the spectral function in the high-temperature phase. At sufficiently high temperatures, quarks and gluons are expected to be good quasiparticles. They lead to a characteristic 'transport peak' $[53,54]$ around $\omega=0$ in the spectral function of some channels; on the other hand, no meson-like excitations are expected, so that the spectral function goes over smoothly to the high-frequency perturbative behavior. The expected behavior is represented by the blue curve in Fig. 2. However, there are strongly-coupled gauge theories where, thanks to the AdS/CFT correspondence, the spectral function can be computed (semi-)analytically [56]. The magenta curve on the figure shows that no transport peak is present and the function $\tanh (\beta \omega / 2) \rho_{i i}(\omega) / \omega^{2}$ is almost constant. This behavior reflects the absence of quasiparticles. Whether the QCD spectral function

\footnotetext{
${ }^{2}$ In Eq. (4.2), real-time evolution is implied, $\hat{J}(t)=e^{i \hat{H} t} \hat{J} e^{-i \hat{H} t}$. The hadronic tensor $W^{\mu v}(q, p)=$ $\frac{1}{2} \sum_{\sigma_{N}} \int d^{4} x e^{i q \cdot x}\left\langle N\left|\hat{J}^{\mu}(x) \hat{J}^{v}(0)\right| N\right\rangle$, which determines the reaction $\gamma^{*}$ Nucleon $\rightarrow X$, is a similar object.
} 

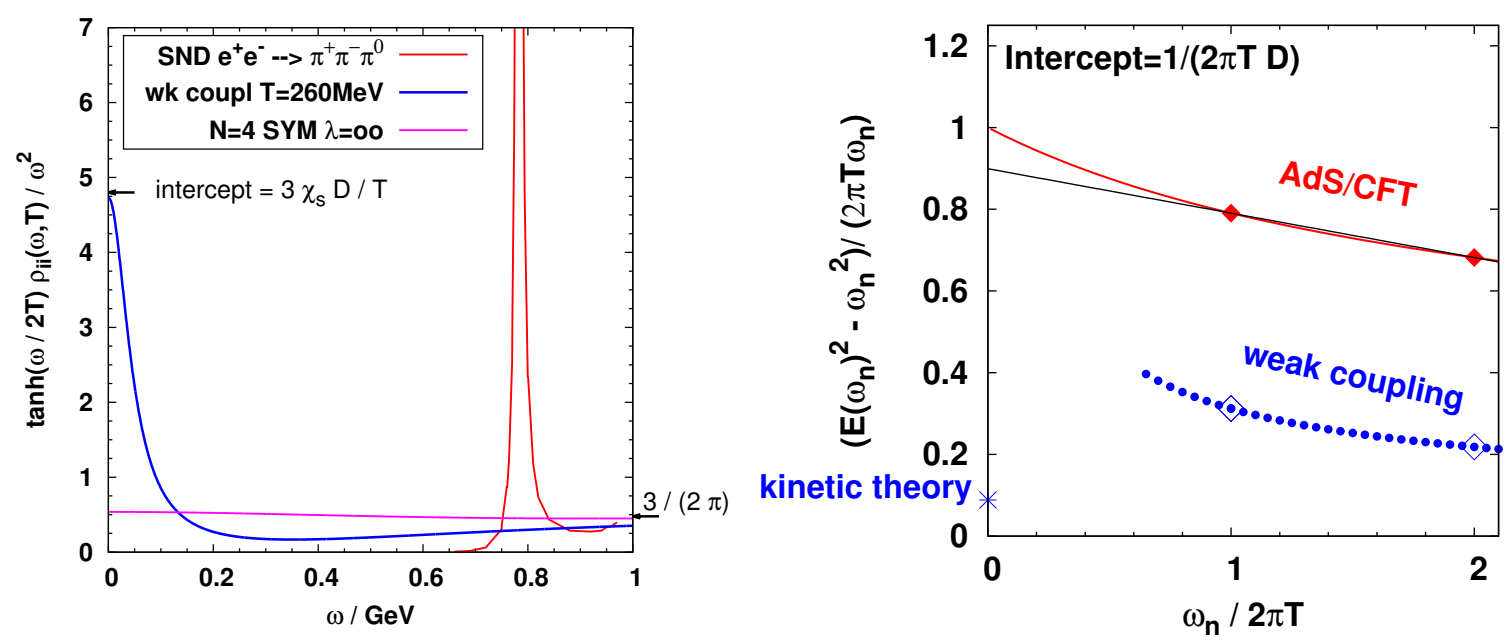

Figure 2: Left: The spectral function of the current $J_{i}=\frac{1}{\sqrt{2}}\left(\bar{u} \gamma_{i} \bar{u}+\bar{d} \gamma_{i} d\right)$ at zero temperature $\left(e^{+} e^{-}\right.$data from [55]) and in the high-temperature phase of QCD at $T=254 \mathrm{MeV}$. In the latter case two 'scenarios' are depicted: one is the spectral function of the $R$-charge current [56] in the large- $N_{c} \mathscr{N}=4$ super-YangMills theory at infinite coupling (multiplied by the factor $\left.\left(3 / N_{c}\right)^{2}\right)$; the other is the QCD weak-coupling prediction, which contains a transport peak [54] due to the existence of quark quasiparticles, and whose large- $\omega$ asymptotic value is $3 /(2 \pi)$. Which scenario is closer to reality at temperatures accessed in heavyion collisions is of central importance to characterize the quark-gluon plasma. Right: The screening mass $E\left(\omega_{n}\right)$ in non-static Matsubara sectors $\omega_{n} \neq 0$. The blue curve corresponds to QCD at weak-coupling at a temperature of $254 \mathrm{MeV}$ [23]. The red curve corresponds to the strongly coupled $\mathscr{N}=4 \mathrm{SYM}$ theory [57, 58]. The continuation of $E\left(\omega_{n}\right)$ down to $\omega_{n}=0$ is expected to yield the inverse diffusion coefficient: the kinetic-theory prediction of [59] is displayed for the weak-coupling case. The black (straight) line going through the first two non-static screening masses directly accessible in the Matsubara formalism shows that a linear extrapolation provides a decent approximation in the strongly coupled case. Both the blue and the red curve tend to 0 for $\omega_{n} \rightarrow \infty$.

at temperatures $200 \lesssim T / \mathrm{MeV} \lesssim 500$ accessed in heavy-ion collisions already admits a transport peak, or instead resembles more a quasiparticle-free spectral function such as the magenta curve is one of the central questions to answer in thermal QCD.

As a related question, one would like to know from what temperature on the weak-coupling shape of the spectral function sets in. It is also not a priori clear at what temperature the $\omega$ and other narrow mesons disappear; they may already have disappeared at $T=155 \mathrm{MeV}$, or instead might survive for a short interval of temperature. The transition to a medium without light mesonlike excitations can also be taken as a definition of deconfinement. For bound states of heavy quarks $(\bar{b} b)$, due to their small size the dissociation temperature is parametrically higher.

Aspects of the inverse problem. - In practice the correlator is known at some discrete points in time, $G\left(x_{0}^{(i)}\right)$. Exploiting the linearity of the problem, we may take linear combinations of Eq. (4.1),

$$
\sum_{i=1}^{n} c_{i}(\bar{\omega}) G\left(x_{0}^{(i)}\right)=\int_{0}^{\infty} d \omega \rho(\omega) \widehat{\delta}(\bar{\omega}, \omega) \equiv \hat{\rho}(\bar{\omega}), \quad \widehat{\delta}(\bar{\omega}, \omega)=\sum_{i=1}^{n} c_{i}(\bar{\omega}) K\left(x_{0}^{(i)}, \omega\right) .
$$

One may now choose the coefficients $c_{i}(\bar{\omega})$ so that the 'resolution function' $\widehat{\delta}(\bar{\omega}, \omega)$ is as narrowly peaked around a given frequency $\bar{\omega}$ as possible. This is the idea behind the Backus-Gilbert method, 
which was recently used in the lattice QCD context [60] (see Refs. therein). One obtains a 'locally smeared' version $\hat{\rho}(\bar{\omega})$ of the spectral function. The optimal resolution function only depends on the kernel and the $x_{0}^{(i)}$; the required coefficients, however, are then wildly oscillating, requiring a regularization. An important feature of the method is that it makes no assumption about the functional form of the spectral function. In fact, in any finite volume the spectral function is a distribution (see Eq. (4.3)), and only $\hat{\rho}(\bar{\omega})$ has a smooth infinite-volume limit [6]. Note that it is in general not true that integrating $\hat{\rho}(\omega)$ with the kernel $K\left(x_{0}, \omega\right)$ yields a good description of the lattice correlator. It is thus also desirable to produce physically reasonable instances of spectral functions which describe the data with a good $\chi^{2}$.

Usually it is assumed that the spectral function can be approximated by a smooth function, e.g. via an explicit ansatz or the maximum entropy method [61]. A new Bayesian method was proposed in [62]; see the talk by S. Kim at this conference. Stochastic methods are being imported from other fields and adapted to lattice QCD; see the talks by H. Ohno and H.-T. Shu.

\subsection{Non-static screening masses}

In this section we review some recent progress in the physics interpretation of screening masses, especially the non-static ones. Consider perturbing the QCD Hamiltonian $\hat{H}$ with an external field $\phi$ coupling to a local QCD operator $\hat{J}$,

$$
\hat{H}_{\phi}(t)=\hat{H}-\int d^{3} y \phi(t, \vec{y}) \hat{J}(t, \vec{y}), \quad \phi(t, \vec{y})=\delta(\vec{y}) e^{\omega t} \theta(-t), \quad \omega \geq 0 .
$$

The latter equation describes a perturbation localized at the origin, starting at $t=-\infty$ and shut off at $t=0$. Let $G_{E}^{J J}\left(\omega_{n}, \vec{x}\right)$ be the Euclidean correlator, taken as a function of Matsubara frequency $\omega_{n}$ and spatial separation $\vec{x}$. Linear response theory leads to the following prediction for the change in the expectation value of the operator $\hat{J}$ due to the external perturbation (see e.g. [6],

$$
\delta\langle J(t=0, \vec{x})\rangle=G_{E}^{J J}\left(\omega_{n}, \vec{x}\right), \quad \text { for } \omega=\omega_{n}=2 \pi T n .
$$

This equation provides the physics interpretation of the static $\left(\omega_{n}=0\right)$ and non-static Euclidean correlators. In particular, the correlation length in Matsubara sector $\omega_{n}$ is the length scale over which a perturbation with the time dependence $e^{\omega_{n} t}$ is screened $(n \geq 0)$. Eq. (4.6) also shows that the dependence of the correlation length on $\omega$ can naturally be continued to continuous values of $\omega$ - even though it is not directly accessible in the Matsubara formalism.

It is well-known that linear response, along with Fick's law $\vec{J}=-D \nabla J_{0}$ as a constitutive equation for the vector current $\vec{J}$, implies the existence of a diffusion pole in the retarded correlator of the charge density $J_{0}[6]$. Let $E\left(\omega_{n}\right)$ be the inverse correlation length of $J_{0}$ in Matsubara sector $\omega_{n}$. In position space, one obtains

$$
G_{E}^{J_{0} J_{0}}\left(\omega_{n}, \vec{x}\right) \stackrel{\omega_{n} \rightarrow 0, \vec{x} \rightarrow \infty}{=}-\chi_{s} E\left(\omega_{n}\right)^{2} \frac{e^{-E\left(\omega_{n}\right)|\vec{x}|}}{4 \pi|\vec{x}|} \quad \text { with } \quad E\left(\omega_{n}\right)^{2} \stackrel{\omega_{n} \rightarrow 0}{\sim} \frac{\omega_{n}}{D}
$$

Thus the hallmark of a diffusion pole is that an external perturbation with the time dependence $e^{\omega t}$ coupling to $J_{0}$ is screened over a distance which diverges as $\sqrt{D / \omega}$ when $\omega \rightarrow 0$. 
Assuming that the diffusion pole exists and is continuously connected to the $n=1$ Matsubara sector, the observation above suggests a new method to extract the diffusion coefficient ${ }^{3}$. If the correlation length can be determined as a function of the Matsubara frequency and continued to small frequencies, then $1 / D$ can be obtained as the slope of the graph of $E\left(\omega_{n}\right)^{2}$ at $\omega_{n}=0$. An advantage of this approach is that at very high temperatures, where $E\left(\omega_{n}\right) \simeq \omega_{n}$ for $\omega_{n} \geq 2 \pi T$, the correct result $1 / D=0$ is obtained; this is in contrast with the method based on the spectral function, where an extremely narrow transport peak would have to be resolved.

This exercise has been carried out [58] in the strongly coupled $\mathscr{N}=4$ SYM theory and shown to reproduce the known result $D=(2 \pi T)^{-1}$ [57]. In QCD at weak coupling, the relevant screening masses have been computed to $\mathrm{O}\left(g^{2}\right)$ [23]; see the right panel of Fig. 2. This calculation is limited to the regime where $\omega_{n}$ is much larger than the Debye mass. On the other hand, the weak coupling result [59] for the diffusion coefficient is also displayed. A turnover of the curve representing the screening masses must occur if it is to make contact with kinetic theory.

Screening masses (static and non-static) in the vector channel have been calculated recently in the high-temperature phase of $N_{\mathrm{f}}=2 \mathrm{QCD}$ on fine lattices [23]. The $\mathrm{O}\left(g^{2}\right)$ predictions for the non-singlet static screening masses at weak coupling were made in [26], and these calculations were extended to the non-static sectors in [23]. The basic physics of these states is that they form non-relativistic bound states of size $\mathrm{O}\left(m_{E}^{-1}\right)$ from the point of view of a $2+1$ dimensional effective theory, where $m_{E}$ is the Debye screening mass. Fairly good agreement is found between the weakcoupling predictions for the screening masses and the lattice results in the non-static Matsubara sector $n=1$. An interesting connection between the weak-coupling calculation of the screening masses and the dilepton production rate [63] was discovered [23], which led to the realization that the same potential entering the latter [64], which was computed non-perturbatively in [65], also determines the spectrum of non-static screening masses. Using this non-perturbatively computed potential improved the agreement with the lattice QCD results. The residues of the screening states in the vector correlation function, which are determined by the wave function of the non-relativistic bound state at the origin, were however found to be significantly larger than the weak-coupling calculations indicates, showing that the states are more tightly bound than at weak-coupling.

\subsection{Numerical studies of spectral functions}

In the following, several channels are discussed where progress has recently been made. I address the channels in what I believe is an increasing order of difficulty. We start with the case of the pion in the low-temperature phase, where chiral effective theory provides theory guidance. Furthermore, the pion parametrically dominates the Euclidean correlators of the axial charge density $\bar{\psi} \gamma_{0} \gamma_{5} \tau^{a} \psi$ and of the pseudoscalar density $\bar{\psi} \gamma_{5} \tau^{a} \psi$.

The pion quasiparticle in the low-T phase.- Consider QCD in the SU(2) chiral limit at temperatures $T<T_{c}$; recall that there must be a sharply defined phase transition, since the chiral condensate $\langle\bar{\psi} \psi\rangle$ is an order parameter. The spontaneous breaking of chiral symmetry implies the presence of a divergent spatial correlation length (Goldstone theorem). Somewhat less obvious is the fact that a massless real-time excitation exists, the pion quasiparticle. At small quark masses $m$, the inverse

\footnotetext{
${ }^{3}$ Since the shear channel also contains a diffusion pole, the same argument applies, with $D$ replaced by $\eta /(e+p)$, $\eta$ being the shear viscosity and $e+p$ the enthalpy density.
} 
spatial correlation length (or 'pion screening mass' $m_{\pi}$ ) is of order $m_{\pi} \sim \sqrt{m}$. The low-momentum dispersion relation of the pion quasiparticle then has the form [66]

$$
\omega_{\vec{p}}=u\left(m_{\pi}^{2}+\vec{p}^{2}\right)^{1 / 2}
$$

with $u$ a temperature-dependent parameter; it is unity at $T=0$ by Lorentz symmetry. In the chiral limit, $u$ corresponds to the group velocity of the pion. Thus a single parameter determines both the quasiparticle mass/screening mass ratio $\omega_{\overrightarrow{0}} / m_{\pi}$ and the $|\vec{p}|$ dependence of the quasiparticle energy.

Exploratory calculations [28] and a more accurate calculation [60] in two-flavor QCD with a zero-temperature pion mass of $270 \mathrm{MeV}$ (at $T=170 \mathrm{MeV}$, which is below $T_{c}$ ) show that $u \approx 0.74$ is significantly smaller than unity. Also, the pion quasiparticle is found to be about $16 \%$ lighter than the zero-temperature pion. This finding contradicts the picture of the hadron resonance gas model, in which the excitations of the medium are taken to be the same as at $T=0$. Recently, evidence was found that the spectral density in the negative-parity nucleon sector also exhibits a significant change below $T_{c}$ [67]. However, these findings are not necessarily inconsistent with the HRG model providing successful predictions for equilibrium quantities [60].

The heavy-quark diffusion constant. - Significant progress has also been made in the study of the diffusion of a heavy quark in the quark-gluon plasma. Heavy-ion collision phenomenology shows that in spite of their heavy mass, charm quarks are 'dragged along' by the medium, so that they too exhibit an azimuthally anisotropic distribution [68]. The 'kicks' of the medium on the heavy quarks therefore have to be strong enough for it to equilibrate.

More precisely, in the static quark limit, the 'kicks' are determined by the color-electric force acting on the worldline of the heavy quark [69]. The autocorrelation of the force ultimately determines the diffusion constant $D$ of the heavy quark via an Einstein relation,

$$
\begin{aligned}
G\left(x_{0}\right) & =\frac{\left\langle\operatorname{Re} \operatorname{Tr}\left(U\left(\beta, x_{0}\right) g E_{k}\left(x_{0}, \overrightarrow{0}\right) U\left(x_{0}, 0\right) g E_{k}(0, \overrightarrow{0})\right)\right\rangle}{-3\langle\operatorname{Re} \operatorname{Tr} U(\beta, 0)\rangle}=\int_{0}^{\infty} d \omega \rho(\omega) K\left(x_{0}, \omega\right), \\
\kappa & =\lim _{\omega \rightarrow 0} T \rho(\omega) / \omega, \quad D=2 T^{2} / \kappa .
\end{aligned}
$$

Here $U\left(t_{\mathrm{f}}, t_{\mathrm{i}}\right)$ is the static quark propagator and $\vec{E}$ the color-electric field. The spectral function has been calculated at next-to-leading order in perturbation theory [70]. It is found to be a smooth function at all frequencies. This represents an advantage for lattice calculations, since resolving narrow features in the spectral function is difficult. On the other hand, the coefficient $\kappa$ has been calculated at NLO [71], and exhibits poor convergence at realistic values of the strong coupling.

Since the early lattice calculations [72], continuum-extrapolated data have been analyzed in quenched QCD [73, 74]. As compared to the NLO calculation, additional spectral weight is required at low frequencies to describe the data. The analysis yields $1.8 \leq \kappa / T^{3} \leq 3.4$. Although phenomenologically, radiative energy loss must be taken into account too, this range is in the right ballpark to explain the spectrum and anisotropic flow of heavy quarks.

The vector channel and the dilepton production rate.- The rate of production of dilepton pairs via virtual photons from a thermal medium is directly proportional to the spectral function $\rho^{\mu}{ }_{\mu}$ of the electromagnetic current $J_{\mu}$ (see e.g. [54] for a derivation). Since the medium produced in heavy-ion collisions appears to be in approximate local thermal equilibrium, a weighted average of the spectrum of dileptons over a range of temperatures is obtained; see for instance [75]. 
We will focus here on correlators of the spatial, non-singlet current $J_{i}$ at vanishing spatial momentum, although preliminary results at non-vanishing spatial momentum on fine, quenched ensembles have been presented by Fl. Meyer at this conference. At zero-temperature, the spectral function $\rho_{i i}$ is dominated by the $\rho$ meson below $\omega=1 \mathrm{GeV}$. The main effect found in recent calculations $[76,77]$ is a shift of the spectral weight from the $\rho$-meson region to the low-frequency region $\omega \lesssim T$ as the temperature increases. The lattice data in the high-temperature phase is consistent with a spectral function containing the sum of a fairly broad transport peak $[76,77,78]$ and the cut contribution $\propto \omega^{2}$; such a spectral function lies somewhere between the two thermal scenarios depicted in Fig. 2. Estimates of the electric conductivity and the diffusion coefficient $D$ have been presented. An important limitation of calculations in dynamical QCD is the lack of a continuum limit of the Euclidean correlator; the continuum limit has been taken in quenched QCD [78]. Even setting this issue aside, uncertainty remains on whether the (infinite-volume) spectral function really is as smooth as the published results suggest.

A scenario where the methods used so far would yield completely incorrect results for the electrical conductivity is if a small fraction of the degrees of freedom contributing to the static susceptibility had a very long mean-free-path; this would be extremely hard to detect in the Euclidean correlator and would lead to a much larger conductivity than the commonly used methods suggest. At the same time, in a relatively fast hydrodynamic evolution of the system, such degrees of freedom would then simply be frozen and not thermalize locally with the rest of the medium. Thus the large diffusion coefficient in this scenario would also be less relevant. It seems implausible to me that such weakly coupled degrees of freedom exist in the high- $T$ phase of QCD.

Kinetic theory, which assumes the existence of weakly interacting quasiparticles, predicts the presence of a transport peak in the spectral function, with an area $\int_{0}^{\Lambda} \frac{d \omega}{\pi \omega} \rho_{i i}(\omega)$ equal to $\chi_{s}\left\langle v^{2}\right\rangle$, where $\left\langle v^{2}\right\rangle$ is the mean-square velocity of the quasiparticles [79] $]^{4}$. If the quarks and gluons are the relevant quasiparticles at some temperature above $T_{c},\left\langle v^{2}\right\rangle \approx 1$ and the spectral weight of the transport peak is known to a reasonable approximation. Thus if a transport peak is resolved and has a spectral weight in the expected range, one can be quite confident in the result. The main challenge at high temperatures is thus to convincingly resolve the transport peak of quark and gluons.

The thermal modification $\Delta \rho(\omega, \vec{k}, T) \equiv \rho(\omega, \vec{k}, T)-\rho(\omega, \vec{k}, 0)$ of the spectral functions in the vector and axial-vector spectral functions is constrained by sum rules. If we define the longitudinal and transverse spectral functions of the isovector vector $V_{\mu}^{a}=\bar{\psi} \gamma_{\mu} \frac{\tau^{a}}{2} \psi$ and axial-vector $A_{\mu}^{a}=\bar{\psi} \gamma_{\mu} \gamma_{5} \frac{\tau^{a}}{2} \psi$ currents via

$$
\begin{aligned}
\frac{1}{3} \int d^{3} x e^{-i \vec{k} \cdot \vec{x}}\left\langle V_{0}^{a}(x) V_{0}^{a}(0)\right\rangle & =\int_{0}^{\infty} d \omega \rho_{V}^{L}(\omega, \vec{k}, T) K\left(x_{0}, \omega\right), \\
-\frac{1}{6}\left(\delta_{i l}-k_{i} k_{l} / \vec{k}^{2}\right) \int d^{3} x e^{-i \vec{k} \cdot \vec{x}}\left\langle V_{i}^{a}(x) V_{l}^{a}(0)\right\rangle & =\int_{0}^{\infty} d \omega \rho_{V}^{T}(\omega, \vec{k}, T) K\left(x_{0}, \omega\right), \\
\frac{1}{3} \int d^{3} x e^{-i \vec{k} \cdot \vec{x}}\left\langle A_{0}^{a}(x) A_{0}^{a}(0)\right\rangle & =\int_{0}^{\infty} d \omega \rho_{\mathrm{A}}^{L}(\omega, \vec{k}, T) K\left(x_{0}, \omega\right)
\end{aligned}
$$

we have the sum rules $[80,28]$,

$$
\frac{1}{2 \pi} \int_{-\infty}^{\infty} d \omega \omega \Delta \rho_{V}^{L}(\omega, \vec{k}, T)=0, \quad \forall \vec{k}
$$

\footnotetext{
${ }^{4}$ The cutoff $\Lambda$ is a separation scale between the inverse mean-free-time and the thermal scale.
} 


$$
\begin{aligned}
\frac{1}{2 \pi} \int_{-\infty}^{\infty} \frac{d \omega}{\omega} \Delta \rho_{V}^{L}(\omega, \vec{k}, T) & =\chi_{s}-\kappa_{l} \vec{k}^{2}+\mathrm{O}\left(|\vec{k}|^{4}\right), \\
\frac{1}{2 \pi} \int_{-\infty}^{\infty} \frac{d \omega}{\omega} \Delta \rho_{V}^{T}(\omega, \vec{k}, T) & =\kappa_{t} \vec{k}^{2}+\mathrm{O}\left(|\vec{k}|^{4}\right), \\
\frac{1}{2 \pi} \int_{-\infty}^{\infty} d \omega \omega \Delta \rho_{\mathrm{A}}^{L}(\omega, \vec{k}, T) & =-\left.m\langle\bar{\psi} \psi\rangle\right|_{0} ^{T},
\end{aligned}
$$

A physics interpretation of $\kappa_{l}$ and $\kappa_{t}$ in terms of screening/antiscreening of electric probe charges and currents placed in the medium exists [27]. These sum rules have been used to constrain the thermal change in the spectral functions. Note that at vanishing spatial momentum, $\rho^{T}=\frac{1}{3} \rho_{i i}$ and Eq. (4.15) simplies to $\int_{0}^{\infty} \frac{d \omega}{\omega} \Delta \rho_{i i}(\omega, T)=0$.

Quarkonium in the high-temperature phase.-The physics of quarkonium in the high-temperature phase has a long history in the heavy-ion community [81]. At what temperature different $\bar{c} c$ and $\bar{b} b$ quasiparticles melt provides a 'thermometer' in heavy-ion collisions: $p$-wave bound states are expected to 'melt' at a lower temperature than $s$-wave bound states. A sequential suppression of the $\Upsilon$ states has been observed in dimuon spectra by CMS [82], providing an interval for the maximum temperature reached in $\mathrm{Pb}+\mathrm{Pb}$ collisions.

The mechanism of the disappearance of $\bar{Q} Q$ quasiparticles has been investigated in detail at weak coupling (see [83] and Refs. therein). The Debye screening of the $\bar{Q} Q$ potential and the scattering with the partons of the medium induce a decrease of spectral weight at treshold in the spectral function of $\bar{Q} \vec{\gamma} Q$ (see Fig. 2 of [84]).

Charmonium quasiparticles have mostly been studied using the full relativistic formulation (e.g. in quenched QCD [85], and in $N_{\mathrm{f}}=2+1$ QCD with heavy Wilson fermions[86]). S-wave charmonium quasiparticles show up in the spectral function up to about $1.4 T_{c}$. We note that the sum rule (4.15) applies in the $J / \psi$ channel and could be used to constrain the thermal changes to the spectral function.

S. Kim presented a study in non-relativistic QCD (NRQCD) at this conference. The pros of using NRQCD are that the exponential kernel $G\left(x_{0}\right)=\int_{-2 m_{Q}}^{\infty} \frac{d \omega}{2 \pi} e^{-\omega x_{0}} \rho(\omega)$ allows for a better $\omega$-resolution; that $\rho(\omega)$ is softer in the ultraviolet than in the relativistic theory; that $\rho(\omega)$ does not contain a transport peak; and that very accurate data can be obtained. All this makes the NRQCD correlator more sensitive to changes in the spectral weight near the $\bar{Q} Q$ threshold. On the downside, dealing with cutoff effects is more delicate in NRQCD.

Bottomomium quasiparticles, due to their large mass, have been addressed using the effective field theories NRQCD and potential NRQCD (pNRQCD). A recent NRQCD calculation [87] in $2+1$ flavor QCD with $m_{\pi}=400 \mathrm{MeV}$ finds that while the ground state $\Upsilon\left(J^{P C}=0^{-+}\right)$survives at least up to $2 T_{c}$, the $\chi_{b_{1}}\left(J^{P C}=1^{++}\right)$melts immediately above $T_{c}$. Ref. [88] on the other hand finds that the $\chi_{b_{1}}$ survives for some interval of temperature above $T_{c}$. Part of the difficulty is that the spectral weight near threshold does not seem to be the dominant one. It would be interesting for future studies to quote the integral of the spectral function over the threshold region, since it is less sensitive to the method used for the inverse problem. Finally, we note that a pNRQCD calculation on the 2+1 flavor ensembles of the HotQCD collaboration has recently emerged [89].

The nucleon channel. - While many bosonic channels have been investigated, the fermionic channels have received very little attention until recently $[67,90]$. One motivation for looking at baryon correlation functions is that they are a probe of chiral symmetry restoration. To be specific, let 
$O_{N+}=\frac{1}{2}\left(1+\gamma_{0}\right) \varepsilon_{a b c}\left(u_{a} C \gamma_{5} d_{b}\right) u_{c}$ be a nucleon interpolating operator (it has an intrinsic positive parity). Consider then its two-point function and corresponding spectral representation [67],

$$
G\left(x_{0}\right)=\int d^{3} x\left\langle O_{N+}(x) \bar{O}_{N+}(0)\right\rangle=\int_{0}^{\infty} \frac{d \omega}{2 \pi} \frac{1}{1+e^{\omega / T}}\left[\rho_{+}(\omega) e^{-\omega x_{0}}-\rho_{-}(\omega) e^{-\omega\left(\beta-x_{0}\right)}\right] .
$$

The restoration of chiral symmetry implies parity doubling: $G\left(\beta-x_{0}\right)=G\left(x_{0}\right)$. Therefore the ratio $R\left(x_{0}\right)=\frac{G\left(x_{0}\right)-G\left(\beta-x_{0}\right)}{G\left(x_{0}\right)+G\left(\beta-x_{0}\right)}$ is an order parameter for chiral symmetry restoration. The same argument applies to static correlators [90], which addresses the degeneracies between screening masses.

Both the static correlators (in a quenched study [90]) and the temporal correlators [67] exhibit the expected behavior that the ratio $R$ starts to drop rapidly at $T=T_{c}$. Approximating the spectral functions $\rho_{+}$and $\rho_{-}$by a single Dirac delta-function $A_{ \pm} \delta\left(\omega-m_{ \pm}\right)$also show that the relative mass difference $\left(m_{-}-m_{+}\right) /\left(m_{-}+m_{+}\right)$drops as $T_{c}$ is approached from below [67]. While little thermal change was found in the positive-parity sector, in principle even the nucleon acquires a thermal width through resonant collisions with the pions of the medium, $N \pi \longleftrightarrow \Delta$.

How strongly baryon quasiparticles are modified below $T_{c}$, and whether any of them survive for a short interval of temperature above $T_{c}$ would be as interesting to know as for the mesons. It may also help understand what happens when a baryon chemical potential is turned on.

\section{Conclusion}

A lot has been learnt about thermal QCD from the lattice. It turns out that the crossover nature of the QCD thermal transition is a very robust feature with respect to varying the quark masses. Efforts to determine the nature of the transition at very small $m_{u d}$ are ongoing. For definitive results, I believe simulating at several lattice spacings $a \lesssim 0.08 \mathrm{fm}$ will be required. For a lattice action with exact chiral symmetry, the requirements can perhaps be relaxed somewhat.

There has been significant progress in the study of near-equilibrium properties via spectral functions. Lattices with $N_{t} \approx 24$ points in the Euclidean time direction have become available, the continuum limit has been taken in quenched QCD and the statistical precision in several channels is now at the permille level. Statistical errors have decreased by an order of magnitude since 2007.

There were many interesting talks about finite-temperature, and indeed about finite-density QCD at this conference, demonstrating the vitality of the field. I thank the organizers for the invitation and for running a very pleasant and informative conference. My own research is supported by the DFG grant ME 3622/2-1 Static and dynamic properties of QCD at finite temperature.

\section{References}

[1] R. Hagedorn, Statistical thermodynamics of strong interactions at high-energies, Nuovo Cim. Suppl. 3 (1965) 147-186.

[2] N. Cabibbo and G. Parisi, Exponential Hadronic Spectrum and Quark Liberation, Phys. Lett. B59 (1975) 67.

[3] A. D. Linde, Infrared Problem in Thermodynamics of the Yang-Mills Gas, Phys. Lett. B96 (1980) 289; E. V. Shuryak, Quantum Chromodynamics and the Theory of Superdense Matter, Phys. Rept. 61 (1980) 71-158. 
[4] J. Engels, F. Karsch, H. Satz, and I. Montvay, High Temperature SU(2) Gluon Matter on the Lattice, Phys. Lett. B101 (1981) 89; L. D. McLerran and B. Svetitsky, A Monte Carlo Study of SU(2) Yang-Mills Theory at Finite Temperature, Phys. Lett. B98 (1981) 195; J. Kuti, J. Polonyi, and K. Szlachanyi, Monte Carlo Study of SU(2) Gauge Theory at Finite Temperature, Phys. Lett. $\mathbf{B 9 8}$ (1981) 199.

[5] N. Brambilla, S. Eidelman, P. Foka, S. Gardner, A. Kronfeld, et. al., QCD and strongly coupled gauge theories: challenges and perspectives, arXiv:1404.3723.

[6] H. B. Meyer, Transport Properties of the Quark-Gluon Plasma: A Lattice QCD Perspective, Eur.Phys.J. A47 (2011) 86, [arXiv:1104.3708].

[7] R. A. Soltz, C. DeTar, F. Karsch, S. Mukherjee, and P. Vranas, Lattice QCD Thermodynamics with Physical Quark Masses, arXiv:1502.0229.

[8] H.-T. Ding, F. Karsch, and S. Mukherjee, Thermodynamics of strong-interaction matter from Lattice QCD, arXiv:1504.0527.

[9] J. I. Kapusta and C. Gale, Finite-temperature field theory: Principles and applications. Cambridge University Press, 2006. 428.

[10] Wuppertal-Budapest Collaboration, S. Borsanyi et. al., Is there still any Tc mystery in lattice QCD? Results with physical masses in the continuum limit III, JHEP 09 (2010) 073, [arXiv:1005.3508].

[11] A. Bazavov, T. Bhattacharya, M. Cheng, C. DeTar, H. Ding, et. al., The chiral and deconfinement aspects of the QCD transition, Phys.Rev. D85 (2012) 054503, [arXiv:1111.1710].

[12] T. Bhattacharya, M. I. Buchoff, N. H. Christ, H. T. Ding, R. Gupta, et. al., The QCD phase transition with physical-mass, chiral quarks, Phys.Rev.Lett. 113 (2014) 082001, [arXiv: 1402.5175$].$

[13] S. Borsanyi, S. Durr, Z. Fodor, C. Holbling, S. D. Katz, S. Krieg, D. Nogradi, K. K. Szabo, B. C. Toth, and N. Trombitas, QCD thermodynamics with continuum extrapolated Wilson fermions II, Phys. Rev. D92 (2015), no. 1 014505, [arXiv: 1504 .0367].

[14] Y. Aoki, G. Endrodi, Z. Fodor, S. Katz, and K. Szabo, The Order of the quantum chromodynamics transition predicted by the standard model of particle physics, Nature 443 (2006) 675-678, [hep-lat/0611014].

[15] HotQCD Collaboration, A. Bazavov et. al., Fluctuations and Correlations of net baryon number, electric charge, and strangeness: A comparison of lattice QCD results with the hadron resonance gas model, Phys.Rev. D86 (2012) 034509, [arXiv: 1203.0784 ].

[16] S. Borsanyi, Z. Fodor, S. D. Katz, S. Krieg, C. Ratti, et. al., Fluctuations of conserved charges at finite temperature from lattice QCD, JHEP 1201 (2012) 138, [arXiv:1112.4416].

[17] S. Borsanyi, Z. Fodor, S. D. Katz, S. Krieg, C. Ratti, and K. K. Szabo, Freeze-out parameters: lattice meets experiment, Phys. Rev. Lett. 111 (2013) 062005, [arXiv:1305.5161].

[18] S. Borsanyi, Z. Fodor, C. Hoelbling, S. D. Katz, S. Krieg, et. al., Full result for the QCD equation of state with 2+1 flavors, Phys.Lett. B730 (2014) 99-104, [arXiv: 1309.5258 ].

[19] HotQCD Collaboration, A. Bazavov et. al., Equation of state in ( 2+1 )-flavor QCD, Phys.Rev. D90 (2014), no. 9 094503, [arXiv:1407.6387].

[20] R. F. Dashen and R. Rajaraman, Narrow Resonances in Statistical Mechanics, Phys. Rev. D10 (1974) 694; R. F. Dashen and R. Rajaraman, Effective Elementarity of Resonances and Bound States in Statistical Mechanics, Phys. Rev. D10 (1974) 708. 
[21] M. Cheng, S. Datta, A. Francis, J. van der Heide, C. Jung et al, Meson screening masses from lattice QCD with two light and the strange quark, Eur.Phys.J. C71 (2011) 1564, [arXiv: 1010.1216 ].

[22] S. Gupta and N. Karthik, Hadronic screening with improved taste symmetry, Phys. Rev. D87 (2013), no. 9 094001, [arXiv:1302.4917].

[23] B. Brandt, A. Francis, M. Laine, and H. Meyer, A relation between screening masses and real-time rates, JHEP 1405 (2014) 117, [arXiv: 1404.2404 ].

[24] B. B. Brandt, A. Francis, H. B. Meyer, O. Philipsen, and H. Wittig, QCD thermodynamics with $O(a)$ improved Wilson fermions at $N f=2$, arXiv:1310.8326.

[25] O. Kaczmarek, E. Laermann, and M. Müller, The thermodynamic and the continuum limit of meson screening masses, PoS LATTICE2013 (2014) 150, [arXiv: 1311 . 3889 ].

[26] M. Laine and M. Vepsalainen, Mesonic correlation lengths in high-temperature QCD, JHEP 02 (2004) 004, [hep-ph/0311268].

[27] B. B. Brandt, A. Francis, and H. B. Meyer, Antiscreening of the Ampere force in QED and QCD plasmas, Phys.Rev. D89 (2014) 034506, [arXiv:1310.5160].

[28] B. B. Brandt, A. Francis, H. B. Meyer, and D. Robaina, Chiral dynamics in the low-temperature phase of QCD, Phys.Rev. D90 (2014) 054509, [arXiv: 1406.5602 ].

[29] L. G. Yaffe and B. Svetitsky, First Order Phase Transition in the SU(3) Gauge Theory at Finite Temperature, Phys. Rev. D26 (1982) 963.

[30] R. D. Pisarski and F. Wilczek, Remarks on the Chiral Phase Transition in Chromodynamics, Phys. Rev. D29 (1984) 338-341.

[31] J. Gasser and H. Leutwyler, Light Quarks at Low Temperatures, Phys.Lett. B184 (1987) 83; J. Bjorken, Highly Relativistic Nucleus-Nucleus Collisions: The Central Rapidity Region, Phys.Rev. D27 (1983) 140-151.

[32] tmfT Collaboration, F. Burger, E.-M. Ilgenfritz, M. P. Lombardo, and M. Müller-Preussker, Equation of state of quark-gluon matter from lattice QCD with two flavors of twisted mass Wilson fermions, Phys. Rev. D91 (2015), no. 7 074504, [arXiv: 1412.6748 ].

[33] A. Francis, O. Kaczmarek, M. Laine, T. Neuhaus, and H. Ohno, Critical point and scale setting in SU(3) plasma: An update, Phys. Rev. D91 (2015), no. 9 096002, [arXiv: 1503.0565 ].

[34] M. P. Lombardo, K. Miura, T. J. Nunes da Silva, and E. Pallante, Chiral symmetry restoration in QCD with many flavours, in 9th Int. Workshop on Critical Point and Onset of Deconfinement (CPOD 2014) Bielefeld, Germany, November 17-21, 2014, 2015. arXiv: 1506.0594.

[35] J. B. Kogut and D. K. Sinclair, The chiral phase transition for lattice QCD with 2 color-sextet quarks, Phys. Rev. D92 (2015), no. 5 054508, [arXiv: 1507.0037 ].

[36] B. Bringoltz and M. Teper, The pressure of the $S U(N)$ lattice gauge theory at large- N, Phys. Lett. B628 (2005) 113-124, [hep-lat/ 0506034 ]; M. Panero, Thermodynamics of the QCD plasma and the large-N limit, Phys. Rev. Lett. 103 (2009) 232001, [arXiv: 0907.3719 ]; S. Datta and S. Gupta, Continuum Thermodynamics of the GluoNc Plasma, Phys. Rev. D82 (2010) 114505, [arXiv:1006.0938].

[37] M. Pepe and U. J. Wiese, Exceptional Deconfinement in G(2) Gauge Theory, Nucl. Phys. $\mathbf{B 7 6 8}$ (2007) 21-37, [hep-lat/ 0610076 ]; G. Cossu, M. D'Elia, A. Di Giacomo, B. Lucini, and C. Pica, G(2) gauge theory at finite temperature, JHEP 10 (2007) 100, [arXiv: 0709.0669 ]. 
[38] M. Bruno, M. Caselle, M. Panero, and R. Pellegrini, Exceptional thermodynamics: the equation of state of $G_{2}$ gauge theory, JHEP 03 (2015) 057, [arXiv: 1409.8305 ].

[39] H. B. Meyer, Glueball matrix elements: a lattice calculation and applications, JHEP 01 (2009) 071, [arXiv:0808.3151]; Y. Chen et. al., Glueball spectrum and matrix elements on anisotropic lattices, Phys. Rev. D73 (2006) 014516, [hep-lat/ 0510074 ]; H. B. Meyer, Glueball Regge trajectories, hep-lat/0508002.

[40] M. Caselle, A. Nada, and M. Panero, Hagedorn spectrum and thermodynamics of SU(2) and SU(3) Yang-Mills theories, JHEP 07 (2015) 143, [arXiv: 1505 . 0110].

[41] H. B. Meyer, High-Precision Thermodynamics and Hagedorn Density of States, Phys. Rev. D80 (2009) 051502, [arXiv:0905.4229].

[42] S. Borsanyi, G. Endrodi, Z. Fodor, S. Katz, and K. Szabo, Precision SU(3) lattice thermodynamics for a large temperature range, JHEP 1207 (2012) 056, [arXiv: 1204.6184$].$

[43] L. Giusti and H. B. Meyer, Implications of Poincare symmetry for thermal field theories in finite-volume, JHEP 1301 (2013) 140, [arXiv:1211.6669].

[44] D. Robaina and H. B. Meyer, Renormalization of the momentum density on the lattice using shifted boundary conditions, PoS LATTICE2013 (2014) 323, [arXiv: 1310 . 6075].

[45] L. Giusti and M. Pepe, Equation of state of a relativistic theory from a moving frame, Phys. Rev. Lett. 113 (2014) 031601, [arXiv:1403.0360].

[46] M. Pepe and L. Giusti, Renormalization of the energy-momentum tensor on the lattice, in Proceedings, 2015 EPS Conference on High Energy Physics, 2015, arXiv: 1510.0451.

[47] K. Kajantie, M. Laine, K. Rummukainen, and Y. Schroeder, The pressure of hot QCD up to $g * * 6$ In(1/g), Phys. Rev. D67 (2003) 105008, [hep-ph/0211321].

[48] C. Gale, S. Jeon, and B. Schenke, Hydrodynamic Modeling of Heavy-Ion Collisions, Int. J. Mod. Phys. A28 (2013) 1340011, [arXiv:1301. 5893]; Y. Akiba et. al., The Hot QCD White Paper: Exploring the Phases of QCD at RHIC and the LHC, arXiv:1502.0273.

[49] X.-Y. Jin, Y. Kuramashi, Y. Nakamura, S. Takeda, and A. Ukawa, Critical endpoint of the finite temperature phase transition for three flavor QCD, Phys. Rev. D91 (2015), no. 1014508 , [arXiv:1411.7461].

[50] H. T. Ding, A. Bazavov, F. Karsch, Y. Maezawa, S. Mukherjee, and P. Petreczky, Chiral phase transition of $N_{f}=2+1 Q C D$ with the HISQ action, PoS LATTICE2013 (2014) 157, [arXiv:1312.0119].

[51] H.-T. Ding, Exploring QCD phase diagram at vanishing baryon density on the lattice, arXiv:1302.5740. [J. Phys. Conf. Ser.432,012027(2013)].

[52] C. Bonati, P. de Forcrand, M. D'Elia, O. Philipsen, and F. Sanfilippo, Chiral phase transition in two-flavor QCD from an imaginary chemical potential, Phys. Rev. D90 (2014), no. 7074030 , [arXiv:1408.5086].

[53] G. Aarts and J. M. Martinez Resco, Transport coefficients, spectral functions and the lattice, JHEP 04 (2002) 053, [hep-ph/ 0203177 ].

[54] G. D. Moore and J.-M. Robert, Dileptons, spectral weights, and conductivity in the quark-gluon plasma, hep-ph/0607172. 
[55] M. N. Achasov et. al., Study of the process $e+e-\longrightarrow$ pi+pi-piO in the energy region $s^{* *}(1 / 2)$ below 0.98-GeV, Phys. Rev. D68 (2003) 052006, [hep-ex/0305049].

[56] R. C. Myers, A. O. Starinets, and R. M. Thomson, Holographic spectral functions and diffusion constants for fundamental matter, JHEP 11 (2007) 091, [arXiv: 0706.0162 ].

[57] P. K. Kovtun and A. O. Starinets, Quasinormal modes and holography, Phys. Rev. D72 (2005) 086009, [hep-th/0506184].

[58] B. B. Brandt, A. Francis, M. Laine, and H. B. Meyer, Vector screening masses in the quark-gluon plasma and their physical significance, Nucl. Phys. A931 (2014) 861-866, [arXiv: 1408.5917 ].

[59] P. B. Arnold, G. D. Moore, and L. G. Yaffe, Photon emission from quark gluon plasma: Complete leading order results, JHEP 0112 (2001) 009, [hep-ph / 0111107 ].

[60] B. B. Brandt, A. Francis, H. B. Meyer, and D. Robaina, The pion quasiparticle in the low-temperature phase of QCD, arXiv:1506.0573.

[61] M. Asakawa, T. Hatsuda, and Y. Nakahara, Maximum entropy analysis of the spectral functions in lattice QCD, Prog. Part. Nucl. Phys. 46 (2001) 459-508, [hep-lat/ 0011040 ].

[62] Y. Burnier and A. Rothkopf, Bayesian Approach to Spectral Function Reconstruction for Euclidean Quantum Field Theories, Phys.Rev.Lett. 111 (2013) 182003, [arXiv:1307.6106].

[63] P. Aurenche, F. Gelis, G. Moore, and H. Zaraket, Landau-Pomeranchuk-Migdal resummation for dilepton production, JHEP 0212 (2002) 006, [hep-ph / 0211036 ].

[64] S. Caron-Huot, $O(g)$ plasma effects in jet quenching, Phys.Rev. D79 (2009) 065039, [arXiv:0811.1603].

[65] M. Panero, K. Rummukainen, and A. Schaefer, A lattice study of the jet quenching parameter, Phys.Rev.Lett. 112 (2014) 162001, [arXiv:1307.5850].

[66] D. T. Son and M. A. Stephanov, Real-time pion propagation in finite-temperature QCD, Phys. Rev. D66 (2002) 076011, [hep-ph / 0204226 ].

[67] G. Aarts, C. Allton, S. Hands, B. Jager, C. Praki, and J.-I. Skullerud, Nucleons and parity doubling across the deconfinement transition, Phys. Rev. D92 (2015), no. 1 014503, [arXiv: 1502.0360 ].

[68] M. Kweon, Recent results on open heavy-flavour observables in relativistic nuclear collisions, Nucl. Phys. A931 (2014) 155-164.

[69] S. Caron-Huot, M. Laine, and G. D. Moore, A way to estimate the heavy quark thermalization rate from the lattice, JHEP 04 (2009) 053, [arXiv: 0901.1195$].$

[70] Y. Burnier, M. Laine, J. Langelage, and L. Mether, Colour-electric spectral function at next-to-leading order, JHEP 08 (2010) 094, [arXiv: 1006.0867 ].

[71] S. Caron-Huot and G. D. Moore, Heavy quark diffusion in perturbative QCD at next-to-leading order, Phys. Rev. Lett. 100 (2008) 052301, [arXiv:0 708 . 4232].

[72] H. B. Meyer, The errant life of a heavy quark in the quark-gluon plasma, New J.Phys. 13 (2011) 035008, [arXiv: 1012 . 0234]; D. Banerjee, S. Datta, R. Gavai, and P. Majumdar, Heavy Quark Momentum Diffusion Coefficient from Lattice QCD, Phys.Rev. D85 (2012) 014510, [arXiv:1109.5738].

[73] O. Kaczmarek, Continuum estimate of the heavy quark momentum diffusion coefficient $\kappa$, Nucl. Phys. A931 (2014) 633-637, [arXiv:1409.3724]. 
[74] A. Francis, O. Kaczmarek, M. Laine, T. Neuhaus, and H. Ohno, A non-perturbative estimate of the heavy quark momentum diffusion coefficient, arXiv:1508.0454.

[75] C. Shen, Z. Qiu, H. Song, J. Bernhard, S. Bass, and U. Heinz, The iEBE-VISHNU code package for relativistic heavy-ion collisions, arXiv:1409.8164.

[76] B. B. Brandt, A. Francis, H. B. Meyer, and H. Wittig, Thermal Correlators in the rho channel of two-flavor QCD, JHEP 1303 (2013) 100, [arXiv: 1212.4200].

[77] G. Aarts, C. Allton, A. Amato, P. Giudice, S. Hands, and J.-I. Skullerud, Electrical conductivity and charge diffusion in thermal QCD from the lattice, JHEP 02 (2015) 186, [arXiv: 1412.6411].

[78] H.-T. Ding, A. Francis, O. Kaczmarek, F. Karsch, E. Laermann, et. al., Thermal dilepton rate and electrical conductivity: An analysis of vector current correlation functions in quenched lattice QCD, Phys.Rev. D83 (2011) 034504, [arXiv:1012.4963].

[79] D. Teaney, Finite temperature spectral densities of momentum and $R$ - charge correlators in $N=4$ Yang Mills theory, Phys. Rev. D74 (2006) 045025, [hep-ph / 0602044 ].

[80] D. Bernecker and H. B. Meyer, Vector Correlators in Lattice QCD: Methods and applications, Eur.Phys.J. A47 (2011) 148, [arXiv:1107.4388].

[81] T. Matsui and H. Satz, J/ $\psi$ Suppression by Quark-Gluon Plasma Formation, Phys.Lett. B178 (1986) 416.

[82] CMS Collaboration, S. Chatrchyan et. al., Indications of suppression of excited $\Upsilon$ states in PbPb collisions at $\sqrt{S_{N N}}=2.76$ TeV, Phys. Rev. Lett. 107 (2011) 052302, [arXiv:1105.4894]; CMS Collaboration, S. Chatrchyan et. al., Observation of sequential Upsilon suppression in $\mathrm{PbPb}$ collisions, Phys. Rev. Lett. 109 (2012) 222301, [arXiv: 1208 . 2826].

[83] M. Laine, How to compute the thermal quarkonium spectral function from first principles?, Nucl.Phys. A820 (2009) 25C-32C, [arXiv: 0810 .1112]; J. Ghiglieri, Review of the EFT treatment of quarkonium at finite temperature, PoS ConfinementX (2012) 004, [arXiv:1303.6438].

[84] M. Laine, A Resummed perturbative estimate for the quarkonium spectral function in hot $Q C D$, JHEP 05 (2007) 028, [arXiv: 0704 .1720].

[85] H. T. Ding, A. Francis, O. Kaczmarek, F. Karsch, H. Satz, and W. Soeldner, Charmonium properties in hot quenched lattice QCD, Phys. Rev. D86 (2012) 014509, [arXiv: 1204 . 4945].

[86] S. Borsanyi et. al., Charmonium spectral functions from 2+1 flavour lattice QCD, JHEP 04 (2014) 132, [arXiv:1401.5940].

[87] G. Aarts, C. Allton, T. Harris, S. Kim, M. P. Lombardo, S. M. Ryan, and J.-I. Skullerud, The bottomonium spectrum at finite temperature from $N_{f}=2+1$ lattice QCD, JHEP 07 (2014) 097, [arXiv:1402.6210].

[88] S. Kim, P. Petreczky, and A. Rothkopf, Lattice NRQCD study of S-and P-wave bottomonium states in a thermal medium with $N_{\mathrm{f}}=2+1$ light flavors, Phys. Rev. D91 (2015) 054511, [arXiv:1409.3630].

[89] Y. Burnier, O. Kaczmarek, and A. Rothkopf, Quarkonium at finite temperature: Towards realistic phenomenology from first principles, arXiv:1509.0736.

[90] S. Datta, S. Gupta, M. Padmanath, J. Maiti, and N. Mathur, Nucleons near the QCD deconfinement transition, JHEP 02 (2013) 145, [arXiv: 1212 . 2927]. 